\title{
Eva Kocher
}

\section{Die Grenzen des Arbeitsrechts}

\section{Der rechtliche Schutz in der Erwerbsarbeit außerhalb von Arbeitsverhältnissen}

\begin{abstract}
„Prekäre“ Arbeit - dieser Begriff wird in Deutschland häufig für jegliche Erwerbsarbeit verwendet, die nicht „Normalarbeit“ im Sinne der für die deutsche Industriearbeit typischen Kern- und Facharbeiterschaft darstellt. Nun ist nicht alles, was nicht normal ist, auch schon prekär und rechtspolitisch abzulehnen; veränderte Geschlechterverhältnisse und veränderte Ansprüche an das Leben müssen und sollen die Erwerbsarbeit schließlich verunsichern. Es gibt aber auch neue Entwicklungen der Unternehmensführung, die durch Verunsicherungen der Beschäftigten über ihren Status und ihre Zukunft zu Prekarität führen. ${ }^{1}$ Und solche Verunsicherungen gibt es nicht mehr nur im Dienstleistungsbereich oder im öffentlichen Dienst; ${ }^{2}$ sie sind in den Sektoren der Industriearbeit angekommen, die als Kernbereiche des westfälischen Modells der Sozialpartnerschaft gelten.
\end{abstract}

\section{Erwerbsarbeit in arbeits- und zivilrechtlichen Verträgen}

\section{Industrielle Dienstleistungen, Outsourcing und Solo-Selbstständigkeit}

Ein Ausdruck dieser Prozesse ist das Wachstum der Dienstleistungsbranchen rund um die Industriearbeit. Die frühere Baufirma Bilfinger Berger macht heute bereits $85 \%$ ihres Umsatzes von $8 \mathrm{Mrd}$ Euro mit Industriedienstleistungen und beschäftigt in diesem Bereich 28.000 Personen. ${ }^{3}$ Diese Branche der „Industrial Services“ wächst schnell. Sie stellt z.B. die Grundlage der „Chemieparks“ dar, Konglomerate unterschiedlichster Dienstleistungs- und Industriebetriebe, die frühere Großbetriebe abgelöst haben: Im Chemiepark Leuna, wo vor der Wende 27.000 Personen in einem einzigen Kombinat beschäftigt waren, arbeiten heute 9.000 Menschen in mehr als 100 Firmen.

Diese Entwicklungen sind lange Zeit unter dem Stichwort „Outsourcing“ diskutiert worden. Bei unternehmerischen Strategien einer Ausgliederung einzelner Funktionen der Wertschöpfungskette werden Tätigkeiten, die vormals durch eigenes Personal erledigt wurden, an Drittunternehmen vergeben. Mittlerweile werden diese Prozesse zunehmend mit dem Begriff des Werkvertrags problematisiert, der das rechtliche Ergebnis bezeichnet: Mit den neu beauftragten (und häufig auch neu gegründeten Unternehmen) schließen Industrieunternehmen in der Regel Werkverträge ab. Nach einer Umfrage der IG Metall vom Februar 2011 sind sich $36 \%$ der befragten Betriebsräte aus 5000 Unternehmen sicher, dass Werkverträge zum Abbau und zur Ersetzung von Stammarbeitsplätzen einge-

1 Zum Begriff der Prekarität siehe insbesondere Schwerpunktheft „Prekarisierung der Arbeit - Prekarisierung im Lebenszusammenhang“ der WSI-Mitteilungen 8/2011 und dort insbesondere die Beiträge von Klenner, Jürgens, Bartelheimer, Schier/Jurczyk/Szymenderski, Klenner/Pfahl/Neukirch/WeßlerPoßberg und Völker.

2 Vogel, Wohlstandskonflikte - soziale Fragen, die aus der Mitte kommen, 2009.

3 Karthaus/Klebe, Betriebsratsrechte Bei Werkverträgen, NZA 2012,417, $418 \mathrm{f}$. 
setzt werden; die Praxis, ganze Produktionslinien durch „Werkverträge“ in der eigenen Halle ausführen zu lassen, hat sich ausgebreitet. ${ }^{4}$

Wo ist hier das Problem? Die Beschäftigten bleiben ja arbeitsrechtlich geschützt; sie sind nur nicht mehr beim Industrieunternehmen selbst, sondern beim Industriedienstleister beschäftigt. Diese Tatsache als solche muss nicht notwendig einen Nachteil darstellen: Man stelle sich vor, ein Hotel beauftrage einen externen Dienstleister mit der Reinigung der Zimmer; für ein angestelltes Zimmermädchen würde dann nur der Tariflohn des Hotelgewerbes gelten, während ein Zimmermädchen bei einem externen Dienst sich auf den Gebäudereiniger-Mindestlohn verlassen könnte. Es dürfte aber kein Zufall sein, dass diese Konstellation bislang nur aus der Theorie ${ }^{5}$ bekannt ist. In der Praxis entsteht ein arbeitsrechtlicher Vorteil solcher Ausgliederungen gerade aus der Möglichkeit, tariflich gewährleistete Bedingungen zu verschlechtern. ${ }^{6}$ In der Regel werden mehr oder weniger personalintensive Arbeiten an Fremdfirmen vergeben, die keiner oder einer niedrigeren Tarifbindung unterliegen.

Diese Praktiken haben in der Industrie dementsprechend erst so richtig Aufschwung genommen, als die Leiharbeit nach Ausschaltung der Dumping-Tarifverträge mit Mindestentgelt und Branchenzuschlägen besser reguliert wurde. Zum Teil handelt es sich auch um verdeckte Leiharbeit und damit rechtlich gesehen um „Scheinwerkverträge“ ${ }^{8}$ Im Januar 2012 ermittelte die Finanzkontrolle Schwarzarbeit in Süddeutschland in sechs Warenzentren der Supermarktketten Kaufland und Netto, die rechtswidrige Werkverträge für Lagerarbeiter und Staplerfahrer abgeschlossen haben, um die Löhne im Lager um rund 30 \% zu drücken. Rossmann hat dafür eigene Subunternehmen gegründet. ${ }^{9}$

Während in den genannten Fällen die Werkverträge mit Unternehmen abgeschlossen werden, die selbst ArbeitnehmerInnen in zumindest rechtlich gesicherten Arbeitsverhältnissen beschäftigen, sind die VertragspartnerInnen in anderen Bereichen - vor allem in der Wissensarbeit - von vornherein nicht arbeitsrechtlich geschützt: Die Zahl der „Solo-Selbstständigen“ ist zwischen 1998 und 2011 um $27 \%$ angestiegen; auch hier sind die Betreffenden überwiegend im Dienstleistungsbereich wie z.B. Hausmeisterdiensten tätig. ${ }^{10}$ Aber selbst in der Automobilindustrie werden Forschungs- und Entwicklungsarbeiten schon bis zu $40 \%$ fremd vergeben, genauso $50 \%$ der EDV-Arbeiten (soweit sie nicht ,intern“ unter den eigenen Beschäftigten „ausgeschrieben“ werden). Eine besondere Erscheinungsform sind die „Crowdworking“-Plattformen, auf denen Unternehmen einzelne IT-Arbeitspakete extern ausschreiben anstatt sie intern zu vergeben. ${ }^{11}$

Was „Selbstständigkeit“ bedeutet, unterscheidet sich sozioökonomisch und beruflich sehr stark; die Betroffenen haben wenig Gemeinsamkeiten und wenig

6 Zu den sonstigen sozioökonomischen Hintergründen ausführlich Däubler, in diesem Heft, S. $133 \mathrm{ff}$.

7 Schüren, NZA 2013, 476; zur Entwicklung genauer Krause, in diesem Heft, S. $119 \mathrm{ff}$.

8 Zur Abgrenzung siehe unten S. 153.

9 Guntram Doelfs, 1,02 Euro pro Schwein, Mitbestimmung 12/2012, 13.

10 Bernhardt, in: Busch/Feldhoff/Nebe (Hrsg.),Übergänge im Arbeitsleben und (Re)Inklusion in den Arbeitsmarkt. Symposium für Wolfhard Kohte, 2012, 113, 114 f.; Bögenhold/Fachinger, Neue Selbständigkeit, 2012, 6, 9 ff.

11 Vgl. z.B. Benner, Mitbestimmung $11 / 2012,61$. 
Verbindung miteinander. ${ }^{12} \mathrm{Zu}$ ihnen zählen die freien Berufe (ÄrztInnen, Steuerberatung, RechtsanwältInnen), die Kultur- und Kreativwirtschaft mit erheblichem Wachstum, Soziale Dienste, Altersbetreuung, pflegerische und medizinische Versorgungsleistungen sowie Beratungsberufe. ${ }^{13}$ Zum Teil kommen die Betroffenen aus der Arbeitslosigkeit, zum Teil wurde ihre „Existenzgründung“ staatlich ge- bzw. befördert. ${ }^{14}$ Das sozialpolitische Problem liegt auf der Hand: $29 \%$ aller Soloselbständigen erhalten ein Einkommen unter 1.100 Euro monatlich und gleichzeitig SGB II-Aufstockung. ${ }^{15}$

\section{Privatrechtliche Verträge der Erwerbsarbeit}

Sozialpolitische Folgen ergeben sich aus der rechtlichen Möglichkeit, die Vertrags- und Beschäftigungsformen zu wählen. Die Wahl zwischen einem „Arbeitsvertrag“ mit „Arbeitnehmer/in“ und „Arbeitgeber/in“ und einem zivilrechtlichen Werk-, Honorar- oder anderen Dienstverträgen mit Auftraggeber/ in und Auftragnehmer/in hat gravierende Rechtsfolgen: Im ersten Fall sind die Regelungen des Arbeitsrechts anwendbar - was zwingende Vorgaben zur Arbeitszeitbegrenzung, zum Mindestentgelt, zum Arbeitsschutz, zur betrieblichen Vertretung sowie zur Interessenvertretung durch Gewerkschaften und in Tarifverträgen zur Folge hat; auch die sozialversicherungsrechtliche Risikosicherung knüpft am Arbeitsverhältnis an. Im zweiten Fall finden Regelungen des allgemeinen bürgerlichen Rechts Anwendung, die vom Grundsatz der Privatautonomie ausgehend nur sehr begrenzt zwingende Vorgaben enthalten.

Die Abgrenzungsfragen zwischen den zivilrechtlichen Vertragsformen der Beschäftigung und dem Arbeitsverhältnis waren schon immer ein rechtspolitisches Problem; es ist lange Zeit unter dem Stichwort „Scheinselbstständigkeit“ diskutiert worden. Es geht hier aber um mehr und anderes als die bloße „Falschbezeichnung“ von abhängig Erwerbstätigen als Selbstständige. ${ }^{16}$ Auch bei den Dreieckskonstellationen der Unternehmens-Werkverträge in SubunternehmerKonstellationen handelt es sich keineswegs durchgehend um Falschbezeichnungen von „Scheinwerkverträgen“. Denn wenn das Entladen eines Schiffes immer schon als Werkvertrag abgewickelt wurde, wieso sollte in bestimmten Fällen nicht auch das Einräumen eines Regals ein „Werk“ darstellen?17

Die Unterscheidung zwischen arbeits- und wirtschaftsrechtlichen Verträgen beruht allerdings auf einer Zuweisung von „Schutz“ und „Verantwortung“, die auf tatsächliche gesellschaftliche Machtverhältnisse reagiert. Insofern lohnt es sich darüber nachzudenken, inwiefern die aktuelle Abgrenzung zwischen arbeitsrechtlichen und wirtschaftsrechtlichen Verträgen der Erwerbsarbeit heutigen tatsächlichen sozialen Bedürfnissen und Grenzen entspricht. Dies soll im Folgenden unternommen werden.

Perulli. Travail économiquement dépendant / parasubordination, 2003. Vgl. auch den Überblick in der Initiativstellungnahme des Europäischen Wirtschafts- und Sozialausschusses von 2010 „Neue Trends bei der selbstständigen Erwerbstätigkeit: der Sonderfall der wirtschaftlich abhängigen selbstständigen Erwerbstätigkeit“, ABl. C 18 vom 19.1.2011, 44-52.

13 Bögenhold/Fachinger (Fn. 13), 15, $20 \mathrm{f}$.

14 Bernhardt (Fn. 13), 114 f. (er hält auch die Novellierung der HandO 2004 für einen wichtigen Faktor, die 53 der 94 Handwerke zulassungsfrei machte); genauso Freedland/Kountouris, in: Davidov/Langille (Hrsg.). The idea of labour law, 2011, 190, 196f. für die Situation in Großbritannien.

15 Bernhardt (Fn. 13), $114 \mathrm{f}$.

16 Das Grünbuch der Europäischen Kommission „Ein modernes Arbeitsrecht für die Herausforderungen des 21. Jahrhunderts“, KOM(2006) 708 endg. bezeichnete dies als "verschleierte Beschäftigung“.

17 Schüren (Interview), Mitbestimmung 12/2012, 18. 


\section{Schutzbedürftigkeit?}

\section{a) Macht und „Schwäche“: Erwerbsarbeit als Existenzsicherung}

Seit das Bundesverfassungsgericht in den Bürgschafts- und HandelsvertreterEntscheidungen anerkannt hat, dass Privatautonomie nur verwirklicht werden kann, wenn das Recht „strukturelle Unterlegenheit“ oder eine „typische Ungleichgewichtslage " berücksichtigt, ${ }^{18}$ sind diese Begriffe auch vermehrt zur Legitimation arbeitsrechtlicher Regelungen herangezogen worden. Sie legen eine Analyse von Macht- und Herrschaftsverhältnissen nahe, die auf die konkreten Vertragsverhandlungen bezogen ist. In der Erwerbsarbeit spielt dabei vor allem eine Rolle, dass Arbeitnehmerinnen und Arbeitnehmer in aller Regel für die Sicherung ihrer wirtschaftlichen und sozialen Existenz auf ein Arbeitsverhältnis angewiesen sind; dies bestimmt ganz wesentlich ihre Verhandlungsposition bei Abschluss eines Arbeitsvertrags. Arbeitsmärkte sind eben im Großen und Ganzen „Käufermärkte“, also Nachfragermärkte, ${ }^{19}$ im Vergleich mit den Exit-Optionen der Arbeitgeberseite sind die Exit-Optionen der Arbeitnehmerseite aus strukturellen Gründen meist überschaubar. ${ }^{20}$

Ein wirkliches Alleinstellungsmerkmal des Arbeitslebens ergibt sich hieraus jedoch nicht. Definiert man „Stärke“ oder „Schwäche“ von Personen in Hinblick auf deren individuelle Ressourcen (ökonomisches Kapital, Bildungskapital, Beziehungskapital etc.), ${ }^{21}$ lässt sich der Schutz von "Schwächeren“ sogar als gemeinsames Prinzip von Arbeitnehmer-, Verbraucher- oder Mieterschutz identifizieren. ${ }^{22}$ Es handelt sich zum Teil sogar um schichttheoretische Überlegungen, wie sie für eine vertragsrechtlich typisierende Anknüpfung nur begrenzt brauchbar sind. Diese sozialpolitischen Aufgaben müssen auch durch Sozialrecht und gesellschaftliche Infrastruktur angegangen werden. ${ }^{23}$

\section{b) Verengung von Optionen im Dauerschuldverbältnis}

Ein spezifisch vertragsrechtliches Ungleichgewicht ergibt sich jedoch daraus, dass sich die Optionen verengen, wenn ein Beschäftigungsverhältnis einmal begründet ist: Beide Seiten legen sich in aller Regel auf eine länger dauernde $\mathrm{Zu}$ sammenarbeit fest. Der Arbeitgeber wird nicht nur die betriebs- und unternehmensspezifischen Qualifikationen, die durch Einarbeitung und manchmal auch durch Qualifizierung entstehen, zu schätzen wissen, sondern auch die Möglichkeit eines Aufbaus stabiler Beziehungen und entsprechender Motivation und Identifikation. ${ }^{24}$ Auf Seiten der ArbeitnehmerInnen wird ohnehin das gesamte Leben meist an betriebliche Anforderungen (Arbeitsort, Arbeitszeit) angepasst, so dass ein Wechsel im Arbeitsverhältnis in aller Regel Rückwirkungen auf das exit- und voice-Optionen siehe Eger/Weise, AuR 1998, 385.

Vgl. zu den Kapitalarten Bourdieu, Ökonomisches Kapital, kulturelles Kapital, soziales Kapital, in: Kreckel (Hrsg.), Soziale Ungleichheiten, 1983, 183 ff.

Hippel, Der Schutz des Schwächeren, 1982.

Eidenmüller, Effizienz als Rechtsprinzip, 2. Aufl. 1998.

Rebhahn, RdA 2009, 154, 163 f. Siehe auch Eger, Eine ökonomische Analyse von Langzeitverträgen, 1995; Jickeli, Der langfristige Vertrag, 1996. 
private Leben hat. Solche oder ähnliche „spezifischen Investitionen“ entstehen jedoch bei jedem Dauerschuldverhältnis. ${ }^{25}$

\section{c) Die Einbindung in eine arbeitsteilige Organisation}

Dennoch greift es zu kurz, die Legitimation arbeitsrechtlichen Schutzes mit der Überlegung anzugreifen, dass hierfür die im allgemeinen Zivilrecht entwickelten Instrumente ausreichen könnten. ${ }^{26}$ Denn es gibt durchaus ein „Alleinstellungsmerkmal“ des Arbeitsverhältnisses: „Das Arbeitsverhältnis ist kein Austauschverhältnis, sondern ein Organisationsverhältnis. Der Großbetrieb will nicht mit tausend einzelnen Leuten Tauschgeschäfte über Arbeitsleistungen abschließen, sondern er braucht die Verfügung über tausend Arbeitskräfte, um sie zu einem Ganzen planmäßig zusammenzuschließen.“ „Es werden nicht Vermögenswerte getauscht, sondern Menschen zu einem Arbeitsverbande geeint. Der Arbeitnehmer [...] schuldet nicht einzelne Arbeitsleistungen, sondern räumt die Verfügung über seine Arbeitskraft ein.“27

Insofern bewirkt der Abschluss eines Arbeitsvertrages, dass die Macht bei Vertragsschluss in Herrschaft verwandelt wird, indem dem Unternehmen per Vertrag das Direktionsrecht $(\$ 106 \mathrm{GewO})$ übertragen wird. Gegenstand des Arbeitsverhältnisses ist mit der „Zurverfügungstellung von Arbeitskraft“ 28 die Person des Arbeitnehmers oder der Arbeitnehmerin. Insofern ist das begründete Schuldverhältnis zwar nicht „personenrechtlich“, aber personenbezogen, denn der Mensch kann bei der Erfüllung des Arbeitsvertrags und der Arbeitsleistung seine „Personenhaftigkeit“ nicht ablegen. ${ }^{29}$ Die Konkretisierungsbefugnis des Arbeitgebers, der die einzelne Arbeitskraft in einen von ihm organisierten Wertschöpfungszusammenhang einbaut, begründet ein Herrschaftsverhältnis.

\section{2. „Persönliche“ Abhängigkeit: Der Rechtsbegriff des „Arbeitnebmers“}

Es ist dieses Herrschaftsverhältnis, das auch rechtlich die Grenzlinie zwischen wirtschaftsrechtlichen Verträgen und Arbeitsrecht bestimmt: Zentralbegriff des Arbeitsrechts ist die „persönliche Abhängigkeit“ im Gegensatz zur „Selbstständigkeit".30

Die Frage nach der „persönlichen Abhängigkeit“ thematisiert, inwiefern der Arbeitnehmer oder die Arbeitnehmerin in die Organisation des Arbeitgeber-Unternehmens eingebunden ist. Die Einbindung wird dabei am Umfang der Weisungsgebundenheit und der Eingliederung in die betriebliche Ablauforganisation gemessen, vor allem hinsichtlich Arbeitszeit und Ausführung der Aufgaben. ${ }^{31}$ Für das Sozialrecht wurde der Kern dieser Rechtsprechung in $\$ 7$ Abs. 1 Satz 2 SGB IV kodifiziert („Anhaltspunkte für eine Beschäftigung sind eine Tätigkeit nach Weisungen und eine Eingliederung in die Arbeitsorganisation des Weisungsgebers"). Im Rechtsvergleich bestehen keine wesentlichen Unterschiede bei diesem wichtigsten Begriff des Arbeitsrechts. ${ }^{32}$ rung auch als Instrument zur Herstellung von beiderseitiger Ko-Investitionsbereitschaft.

26 So aber Schäfer, in: Sadowski/Walwei (Hrsg.). Die ökonomische Analyse des Arbeitsrechts, 2001, 23.

27 Potthoff, Wesen und Ziel des Arbeitsrechts, 1922, 18; $38 \mathrm{ff}$.

28 So auch die ständige Rechtsprechung des BAG (BAGE 109, 87; BAGE 125, 257: „Die Leistungspflicht [...] orientiert sich an der Leistungsfähigkeit des Arbeitnehmers").

29 Schwerdtner, Fürsorgetheorie und Entgelttheorie im Recht der Arbeitsbedingungen, 1970, $86 \mathrm{f}$. Siehe z.B. aus der neueren Literatur Breinlinger, in: Festschrift für Klaus Bepler, 2012, 27.

30 BAG 20.10.1993, AfP 1994, 72.

31 Siehe Preis, in: ErfK, 12. Aufl. 2012, \611 BGB Rn. 50 ff.

32 Genauer Nogler, ZESAR 2009, 461, 464 f.; Wank, Auk 2007, 244, 246 f; Rebhahn (Fn. 27), 155 f. 
Die Abgrenzungsfragen waren schon immer ein praktisches und politisches Problem. Zur Verhinderung von Umgehung empfiehlt die ILO-Empfehlung 198 betreffend das Arbeitsverhältnis ${ }^{33}$ in Nr. 11 Instrumente, wie sie auch im deutschen Recht entwickelt und erprobt wurden. Am wichtigsten ist dabei die Verwendung eines „Typus“-Begriffs. Die Arbeitsgerichte (sowie der daran anknüpfende Gesetzgeber des $₫ 7$ Abs. 1 SGB IV) bedienen sich dabei „nicht des tatbestandlich scharf kontrollierten Begriffs, der auf eine einfache Subsumtion hoffen ließe, sondern der Rechtsfigur des Typus; die [erfassten...] Personen werden nicht im Detail definiert, sondern ausgehend vom Normalfall in der Form eines Typus beschrieben. [...] Maßgeblich ist das Gesamtbild“, ${ }^{34}$ Letztlich wird dabei eine „wirtschaftliche Betrachtungsweise“ geübt, die den „Vorrang der Tatsachen " festschreibt und damit Umgehung erschwert: ${ }^{35}$ Die Einordnung wird nicht durch die Bezeichnung und Beschreibung des Vertrags durch die Parteien bestimmt, sondern durch die tatsächlich gelebte Praxis.

Der Typus bleibt aber immer auf die persönliche Abhängigkeit, also auf die Einbindung in die unternehmerische Arbeitsorganisation, bezogen.

\section{Existenzsicherung durch Erwerbsarbeit auf dem Markt}

$\mathrm{Da}$ es Schutzbedürfnisse auch außerhalb von organisatorischen Einbindungen gibt ${ }^{36}$ kennt bereits das geltende Recht arbeitsrechtliche Normen und Vorgaben für zivilrechtliche Verträge.

\section{a) Regelungen für „abhängige Selbstständigkeit“}

Im Graubereich zwischen Arbeits- und Zivilrecht ist es insbesondere das Konzept der „abhängigen Selbstständigkeit“ in der Erwerbsarbeit, das zumindest einen Teil der „neuen Selbstständigkeit“ von „Kleinstunternehmern“ und „Soloselbständigen“ durch zwingende Schutzvorschriften erfassen kann. In zahlreichen europäischen Staaten gibt es einen solchen Status zwischen Arbeitsvertrag und Selbstständigkeit: In Spanien werden sie durch Regelungen für "wirtschaftlich abhängige Beschäftigte” geregelt, ${ }^{37}$ in Italien gibt es Schutz für die in "parasubordinazione" Beschäftigte und Projektbeschäftigte, ${ }^{38}$ das Arbeitsrecht des Vereinigten Königreichs kennt neben dem “employee” die Kategorie der “worker”, ${ }^{39}$ und im deutschen Recht wird mit Hilfe des Begriffs der „arbeitnehmerähnlichen Person“ der Anwendungsbereich bestimmter arbeitsrechtlicher Schutznormen auf „wirtschaftlich abhängige “ Personen erweitert. ${ }^{40}$

Die Reichweite des Schutzes ist jedoch ganz unterschiedlich geregelt. Zum Teil wird für die abhängigen Selbstständigen lediglich sozialversicherungsrechtlicher Schutz vorgesehen. Überwiegend werden darüber hinaus zwar auch die vertrag-

Zu Hintergrund und Entwicklung genauer Freedland/Kountouris, The Legal Construction of Personal Work Relations, 2011, $23 \mathrm{f}$.

34 BVerfG, AP Nr. 82 zu $\$ 611$ BGB Abhängigkeit, Rn. 7.

35 Waas, Werkvertrag, freier Dienstvertrag und Arbeitsvertrag: Abgrenzung und Identifikation im deutschen Recht und in ausländischen Rechtsordnungen, 2012.

36 Siehe z.B. Davidov, in: Fudge/McCrystal/Sankaran (Hrsg.). Challenging the legal boundaries of work regulation, 2012,171, $179 \mathrm{f}$ zur Unterscheidung zwischen „subordination“ und "dependency “ und zum Begriff „freelancer“.

37 Vgl. auch Soravilla/Herrezuelo, EuzA 2010, 127; Landa Zapirain, in: Fudge/McCrystal/Sankaran (Hrsg.) (Fn. 39), 2012, 155.

38 Ausführlich Borzaga, in: Busch/Feldhoff/Nebe (Hrsg.). (Fn. 10), 2012, 99; Nogler, The concept of "subordination" in European and comparative law, 2009, $88 \mathrm{ff}$.

39 Zum englischen Recht siehe z.B. Nogler (Fn. 38), 117 ff. Ein Überblick über diese Regelungen findet sich schon in der Initiativstellungnahme des Europäischen Wirtschafts- und Sozialausschusses (Fn. 12) vom 19.1.2011; siehe auch Waas (Fn. 35), dort auch zum schwedischen und österreichischen Recht. 
lichen Beziehungen zum Kunden oder der Kundin geregelt; der Gegenstand der Regulierung (Mindesteinkommen, Arbeitszeit, Kündigungsschutz, Zugang zu den Arbeitsgerichten, Urlaub etc.) unterscheidet sich von Rechtsordnung zu Rechtsordnung jedoch erheblich. Hier ist Raum für Experimente, der in Deutschland noch lange nicht ausgeschöpft ist. ${ }^{41}$

Der Tatbestand der Schutzbedürftigkeit wird in aller Regel durch zwei Merkmale definiert, deren Verhältnis zueinander alles andere als klar ist: Vorausgesetzt wird in aller Regel, dass der/die Erwerbstätige

- die Leistung persönlich und ohne eigene Beschäftigte erbringt, und/oder

- an einen einzigen Kunden oder eine einzige Auftraggeberin gebunden ist. ${ }^{42}$ Diese „wirtschaftliche Abhängigkeit“ wird z.T. an der Höhe des Umsatzes aus der für diesen Kunden geleisteten Arbeit, z.T. an der Dauer der Beziehung zwischen Erwerbstätigem und Kunden gemessen. ${ }^{43}$

Das zweite Merkmal (wirtschaftliche Abhängigkeit von einem einzigen Unternehmen und Auftraggeber) ist durch das Fehlen von exit-Optionen begründet; auch bestehen häufig Einbindungen und Abstimmungen organisatorischer Art (z.B. bei der Vernetzung der Produktion durch „just-in-time“-Konzepte). Wirtschaftliche Abhängigkeit in diesem Sinn erfordert jedenfalls einen gewissen Kontinuitätsschutz (= Kündigungsschutz) und Schutz vor willkürlichem Umgang. Solche Abhängigkeiten gibt es jedoch im Wirtschaftsleben in vielfacher Form, nicht nur in Erwerbsarbeitsverträgen, sondern auch in anderen Langfristbeziehungen zwischen Unternehmen wie z.B. langfristigen Lieferbeziehungen oder den Abhängigkeiten zwischen Zuliefer- und Abnehmerunternehmen.

Im Gegensatz dazu bezieht sich das erste Merkmal („Solo“-Selbstständigkeit) auf ein grundlegendes Merkmal abhängiger Erwerbsarbeit: Die Arbeitskraft, die Vertragsgegenstand ist, lässt sich nicht von der Person trennen. Daraus erwachsen Gefahren für die Person (Gesundheit und Sicherheit), die Vereinbarkeit von Privatleben und Erwerbsleben sowie Notwendigkeiten der langfristigen Sicherung sozialer Risiken und der sozialen Kooperation.

Diese Gefahren der Solo-Selbstständigkeit bestehen jedoch unabhängig von der wirtschaftlichen Abhängigkeit. Auch wenn eine Person für eine Reihe unterschiedlicher Auftraggeberinnen und Auftraggeber Erwerbsarbeit im Rahmen zivilrechtlicher Verträge ausübt, kommt sie in der Vertragsdurchführung an die Grenzen der Person. Die Verhandlungsposition wird auch in solchen Konstellationen dadurch beeinflusst, dass die Arbeitskraft notwendige körperliche Grenzen hat. Zum sozialen Problem wird dies durch die grundsätzliche Abhängigkeit von der Erwerbsquelle „Arbeitskraft“ - mit „wirtschaftlicher Abhängigkeit“ in dem Sinne, wie es die Gesetze zur abhängigen Selbstständigkeit beschreiben, hat dies jedoch nicht notwendig etwas zu tun.

Dieses Merkmal der „Solo-Selbstständigkeit“ spricht dafür, für die Erwerbsarbeit auch in zivilrechtlichen Vertragsformen geeignete Schutzkonzepte zu entwickeln.

41 Siehe dazu auch Däubler, in diesem Heft, S. $133 \mathrm{ff}$.

42 Siehe z.B. \$12a TVG aus dem deutschen Recht; sowie die Rechtsprechung: BAG 17.10.1990, BAGE 66, 113 (116); BAG 8.9.1997, AP Nr. 38 zu $\ 5$ ArbGG; BAG 16.7.1997, AP Nr. 37 zu $\$ 5$ ArbGG; BAG 11.4.1997, AP Nr. 30 zu $\ 5$ ArbGG; ebenso BGH 4.11.1998, NZA 1999, 53.

43 Initiativstellungnahme des Europäischen Wirtschafts- und Sozialausschusses (Fn. 12) vom 19.1.2011; siehe z.B. die italienischen Kriterien der Dauer der Beziehung sowie der „Koordination“ zwischen Arbeitsaktivität und betrieblichen Zielen (Borzaga (Fn. 41), 100 f.). 
Sucht man hierfür nach Vorbildern aus dem geltenden Recht, so erscheint der Begriff der „Beschäftigung“ nur auf den ersten Blick geeignet. Er wird im deutschen Recht als Oberbegriff für unterschiedliche Rechtsformen von Erwerbsarbeitsverhältnissen verwandt; meistens geht es dabei jedoch um die Erweiterung des Anwendungsbereichs arbeitsrechtlicher Gesetze auf Beamtinnen und Beamte, manchmal auch Auszubildende, arbeitnehmerähnliche Personen oder Bewerberinnen und Bewerber. ${ }^{44}$

Ergiebiger sind Regelungen, die sich explizit auf die selbstständige Erwerbsarbeit beziehen. Hier wird meist vor allem die soziale Sicherung vor Risiken thematisiert; insbesondere eine Rentenversicherungspflicht wäre sinnvoll, da sie die wirklichen Kosten der Arbeitskraft auf dem Markt transparenter machen könnte. ${ }^{45}$ Diese Formen der Risikoabsicherung sind allerdings nicht vertragsbezogen, sondern personenbezogen.

Vertragsrechtlich interessant sind hingegen Regelungen über die Anwendung des Arbeitsschutzrechts als Ausfluss allgemeiner vertraglicher Nebenpflichten außerhalb der „abhängigen“ Erwerbsarbeit. Hier ist aus dem deutschen Recht insbesondere auf die allgemeine Fürsorgepflicht des $₫ 618$ BGB hinzuweisen; sie ist in einem werkvertraglichen Verhältnis analog anzuwenden, wenn der Unternehmer zur Erfüllung der ihm obliegenden Verrichtungen die Räume des Bestellers betreten muss. ${ }^{46}$ Die Europäische Bildschirmrichtlinie 90/270/EG ist nach der Rechtsprechung des EuGH auf alle Arbeitsplätze anwendbar, unabhängig davon, ob sie mit „Arbeitnehmern“ besetzt sind. ${ }^{47}$ Regelungen der Arbeitszeiten von selbstständigen Kraftfahrern verfolgen auch Arbeitsschutzziele. ${ }^{48}$ Die Antidiskriminierungsvorschriften erfassen in der Regel den Zugang zur selbständigen Erwerbstätigkeit. ${ }^{49}$ Noch weiter geht die Richtlinie 2010/41/EU über den Mutterschutz für Frauen, die eine selbständige Erwerbstätigkeit ausüben. ${ }^{50}$ Sie verlangt nicht nur sozialrechtliche Leistungen, sondern auch Vertretungslösungen; an eine Verantwortungszuweisung an Auftraggeber ist jedoch nicht gedacht. Am interessantesten wird es in Hinblick auf Rechte zur kollektiven Organisation: Steht den Milchbauern und neuen Selbstständigen ein Streikrecht zu? Aus Art. 9 Abs. 3 GG dürfte sich dies jedenfalls entnehmen lassen. ${ }^{51}$

Die rechtspolitische Diskussion mag hier noch weniger fortgeschritten sein als im Fall der „arbeitnehmerähnlichen Personen“. Für die Beantwortung der Frage

\4 Abs. 1 BPersVG, $\ 4$ Abs. 1 BGleiG, $\ 2$ Abs. 2 ArbSchG, $\$ 7$ Abs. 1 PflegeZG, $\$ 6$ Abs. 1 AGG; vgl. auch $₫ 2$ Abs. 2 Nr. 1 SGB IV, wo $\$ 7$ SGB IV jedoch klarstellt, dass hier nichts anderes gemeint ist als „Arbeitnehmer“. Kritik an der uneinheitlichen Verwendung (Verstoß gegen das Transparenzgebot) bei Richardi, NZA 2010, 1101.

45 Für einen Überblick im deutschen Recht siehe Bernhardt (Fn. 10); zur Einbeziehung in die gesetzliche Rentenversicherung (und Streichung des $\$ 2$ S. 1 Nr. 9b SGB VI) siehe Beschluss 13 der Abteilung Arbeits- und Sozialrecht des 68. DJT $2010 \mathrm{im}$ Anschluss an Waltermann. Verhandlungen des 68. Deutschen Juristentages Berlin 2010. Bd. 1: Gutachten, 2010, B1-B120.

46 BGHZ 5, 62; siehe schon RGZ 159, 268.

47 EuGH 12.12.1996 - C-74/95 u C-129/95, NZA 1997, 307.

48 Gesetz zur Regelung der Arbeitszeit von selbständigen Kraftfahrern v. 11.7.2012, das insofern Richtlinie 2002/15/EG umsetzt (siehe aber Wiebauer, NZA 2012, 1331, der die Notwendigkeit des Arbeitsschutzes von Selbstständigen bestreitet).

49 Richtlinien 2000/43/EG, 2004/113/EG und 2006/54/EG, im deutschen Recht umfassender $\$ 6$ Abs. 3 AGG.

50 Im Vorschlag der Kommission war noch ein Recht auf Urlaub zur Pflege von Familienangehörigen vorgesehen (KOM(2008)636 endg).

51 Schlachter, in: Gedächtnisschrift für Ulrich Zachert, 2010, 634, 639, 643 ff.; sie weist auch auf das ILOÜbereinkommen Nr. 141 über die Verbände ländlicher Arbeitskräfte hin, das selbstständig Tätige erfasst. Siehe auch ebd., $640 \mathrm{f}$. zur Abgrenzung anhand von Kriterien des jeweiligen Marktes: Vielzahl "kleiner" Leistungsanbieter, deutlich geringere Zahl potenzieller Vertragspartner, Leistungen gegeneinander austauschbar, Leistungsanbieter überwiegend allein tätig. 
„Braucht man einen Grundstock an Vorschriften, welche die Beschäftigungsbedingungen aller Beschäftigten, unabhängig von der Form ihres Vertrags, regeln?" 52 bedürfte es aber zunächst der Anerkennung, dass es im allgemeinen $\mathrm{Zi}$ vilrecht „Verträge über Arbeit“ gibt, die unabhängig von persönlicher oder wirtschaftlicher Abhängigkeit der Regelung bedürfen.

\section{Die Verantwortung der Unternehmen als Arbeitgeber}

In der Folge wird die Frage der Verantwortlichkeit und Verpflichtungen immer virulenter: Gegen welche ökonomische Einheit können grundlegende Rechte der Erwerbsarbeit denn durchgesetzt werden ${ }^{53}$

\section{Das Abgrenzungsproblem in Dreieckskonstellationen}

Dies ist das Kernproblem der Dreieckskonstellationen. In der Grundform schließt eine Beschäftigte oder ein Beschäftigter einen Arbeitsvertrag mit einem „Subunternehmen“ ab, das wiederum einen Werkvertrag mit dem Hauptauftraggeber abschließt - der wiederum die Arbeitskraft der ArbeitnehmerInnen indirekt nutzt. Ähnliche Dreieckskonstellationen entstehen bei Leiharbeit, Outsourcing und allgemein insbesondere bei der Nutzung von Industriedienstleistungen (Werk-, Dienst- oder Personalgestellungsverträge). ${ }^{54}$

Auch hier stehen zunächst die Abgrenzungsfragen im Vordergrund, denn durch die Dreieckskonstellation ergeben sich zahlreiche Missbrauchs- und Umgehungsgefahren. ${ }^{55}$ Subunternehmerverträge dienen häufig der Verschleierung von illegaler Arbeitnehmerüberlassung. ${ }^{56}$ Für die Abgrenzung zwischen Werkvertrag und Leiharbeit/ „Scheinwerkvertrag“ gibt es eine Reihe von Kriterien, ${ }^{57}$ wobei auch hier die Frage, wer das Weisungsrecht tatsächlich ausübt, eine zentrale Rolle spielt; die Direktionsbefugnis stellt nun einmal den Kern der „persönlichen Abhängigkeit“ und damit des Arbeitsvertrags dar. Allerdings: Auch der Werkbesteller darf natürlich Einfluss auf die Herstellung des Werks nehmen, und eine häufige Kontrolle kann „sowohl Arbeitnehmerüberlassung als auch ein gutes Qualitätsmanagement indizieren.“ Etwas trennschärfer mag die Abstellung auf die Gefahrtragung und Mängelgewährleistung sein. ${ }^{58}$ Und hier muss es natürlich ebenfalls „wirtschaftlich“ auf den „Typ“ und damit die tatsächliche Vertragsdurchführung ankommen. ${ }^{59}$

\section{Aufspaltung der Verantwortung bei Aufspaltung von Arbeitgeberfunktionen?}

Nicht selten führt Outsourcing durchaus zu „echten“ Werkvertragskonstellationen. Für das Arbeitsrecht ist es jedoch wie für andere einseitig zwingende Ordnungen kein grundsätzlich fremder Gedanke, über die formalrechtliche An-

Grünbuch zum Arbeitsrecht (Fn. 19), KOM(2006) 708 endg, Frage 8.

Vgl. auch Hyde, in: Fudge/McCrystal/Sankaran (Hrsg.) (Fn. 39), 2012, 83, 93 f.

Veneziani, in: Hepple/Veneziani (Hrsg.). The transformation of labour law in Europe. A comparative study of 15 countries, 1945-2004, 2009, 99, $118 \mathrm{ff} . ;$ vgl. Grünbuch zum Arbeitsrecht (Fn. 16), $\mathrm{KOM}(2006) 708$ endg. („dreiseitige Rechtsverhältnisse“) (Frage 9).

Fink, Der Arbeitgeberwechsel als Möglichkeit zur Tarifflucht, 1999, $180 \mathrm{ff}$.

Weber, Das aufgespaltene Arbeitsverhältnis, 1992, $62 \mathrm{ff}$.

Weber (Fn. 59), 63 f; Schüren, in: Festschrift für Wolfgang Däubler, 1999, 90; LAG Berlin 1.9.1989, LAGE Nr. 2 zu $₫ 611$ BGB Arbeitgeberbegriff. Zum Referentenentwurf von 1975 mit seinen Abgrenzungskriterien siehe auch Becker, ZIP 1981, 699, 706; siehe auch aktuell Positionspapier des DGB: www.dgb.de/-/5X9.

Schüren (Fn. 60), 93 f., $98 \mathrm{f}$.

BAG 18.1.2012, NZA-RR 2012, 455. 
knüpfung an einem Vertrags-Arbeitgeber hinauszudenken. Das Bundesarbeitsgericht hat einmal als allgemeinen Grundsatz formuliert: „Bieten sich dem Arbeitgeber verschiedene arbeitsvertragliche Gestaltungsformen an, die für den Arbeitnehmer zu einem unterschiedlichen arbeitsrechtlichen Schutz führen, darf er nicht willkürlich die ihm günstigere auswählen. "60 Thomas Blanke hat ein arbeitsrechtliches „Instrumentalisierungsverbot“ vorgeschlagen: Der Schutz des „Normalarbeitsverhältnisses“ dürfe nicht durch den Rückgriff auf „atypische Beschäftigung“ gezielt unterlaufen werden. ${ }^{61}$

Der zitierte Satz des Bundesarbeitsgerichts stammt aus dessen Rechtsprechung zur „mittelbaren“ Arbeitgeberstellung. ${ }^{62}$ Hier interpretierte es die Arbeitgeberstellung „wirtschaftlich“: „Arbeitgeber“ sei derjenige, der unternehmerische Entscheidungen treffen und Gewinn erzielen könne. Auch wenn das Gericht bislang eine mittelbare Arbeitgeberstellung nur bei der Zwischenschaltung von Arbeitnehmerinnen oder Arbeitnehmern (also natürlichen Personen) in Betracht gezogen hat: ${ }^{63}$ Die Überlegung einer arbeitsrechtlichen Verpflichtung desjenigen Unternehmens, das die Arbeitskraft tatsächlich für seine Wertschöpfung nutzt, neben oder anstelle des Vertragsarbeitgebers erscheint grundsätzlich als überzeugender Ansatz. ${ }^{64}$

Allerdings dürfte die Suche nach dem „wirklichen Arbeitgeber“ in Wertschöpfungsketten mit komplizierten Abhängigkeitsverhältnissen unter den Unternehmen häufig leer laufen. Bei solchen Aufspaltungen von Arbeitgeberfunktionen ist eher an eine Aufspaltung und Vermehrung der Verantwortlichkeiten zu denken (vgl. auch die Begriffe „partielles Arbeitsverhältnis“, ${ }^{5}$ „Co-Arbeitgeber“66 oder „funktioneller Arbeitgeberbegriff“). ${ }^{67}$ Beispiele für eine solche wirtschaftliche Betrachtungsweise finden sich in Vorbildern für einen „arbeitsrechtlichen Durchgriff“ “ ${ }^{68}$ Er hat hauptsächlich in Haftungsfällen Bedeutung, in denen der Missbrauch formeller Rechtsstellungen durch die Berufung auf die Selbständigkeit einer juristischen Person durch eine wirtschaftliche Sichtweise durchbrochen wird. ${ }^{69}$ Im positiven Recht gibt es hierfür Vorbilder bei der vorübergehenden Haftung des Betriebsübergebers nach $\$ 613$ a Abs. 2 BGB, der Haftung des Entleihers in $\mathbb{\$} 10$ Abs. 1, $\mathbb{\int} \ 13-13 \mathrm{~b}$ AÜG oder der Haftung des Auftraggebers für den Subunternehmer nach $\$ 14$ AEntG.

Von einer gewissen Bedeutung sind mittlerweile auch wirtschaftsrechtliche Verträge, in denen Arbeitgeberverantwortung zugewiesen wird. So übernehmen Unternehmen z.T. im Rahmen ihrer CSR-Politiken ${ }^{70}$ vertraglich die Verantwortung für Zulieferunternehmen. ${ }^{71}$ Und faktisch beherrschende Unternehmen werden politisch als "super-employers" der Beschäftigten in den Zulieferunterneh-

BAG 20.7.1982, BAGE 39, 200.

Blanke, Thesen zur Zukunft des Arbeitsrechts, KJ 2004, $11 \mathrm{ff}$.

BAG 20.7.1982, BAGE 39, 200.

BAG 9.4.1957, AP Nr. 2; BAG 8.8.1958, AP Nr. 3; BAG 26.11.1975, AP Nr. 19, jeweils zu $\$ 611$ BGB Mittelbares Arbeitsverhältnis; BAG 20.7.1982, AP Nr. 5 und 9.9.1982, AP Nr. 1 zu $\ 611$ BGB Hausmeister. Weitere Fälle bei Weber (Fn. 56), 85 ff. Vgl. BAG 11.9.1991, AP NR. 29 zu Internationales Privatrecht Arbeitsrecht (Goethe-Institut). Fink (Fn. 55), 182 f. hält dies für keineswegs zwingend. Siehe auch Fink (Fn. 58), 182 f.

Siehe Däubler, in diesem Heft S. $133 \mathrm{ff}$.

Supiot, Beyond Employment, 2001.

Fabricius, Rechtsprobleme gespaltener Arbeitsverhältnisse im Konzern, 1982, 27 ff. zur Lehre vom "funktionellen Arbeitgeberbegriff" von Nikisch und Hueck. Siehe BSGE 18, 190. Siehe auch BAG 19.7.1957, AP Nr 1 zu $\$ 1$ GesamthafenbetriebsG (zum Gesamthafenbetrieb als funktioneller Arbeitgeber).

Fink (Fn. 58), $186 \mathrm{ff}$.

Hyde (Fn. 56), 93 ff. zu den Konzepten „respondeat superior“ und „vicarious liability“, mit Beispielen aus dem US-amerikanischen Recht. Siehe aber auch BAG 9.4.1987, BAGE 55, 206.

Siehe zu Konzept und Kritik schon Kocher, KJ 2010, 29.

Hyde (Fn. 56), 97 ff. 
men verantwortlich gemacht, ${ }^{72}$ wie es aktuell und $\mathrm{zu}$ Recht das Unternehmen

Kik wegen des Brands in einem Zulieferbetrieb in Pakistan erlebt hat.

In der Tarifpolitik spielt solche Verantwortungszuweisung vor allem im Verhältnis zur Leiharbeit eine Rolle. So regeln die jüngsten tariflichen Regelungen in der Stahlindustrie auch Verpflichtungen der Entleihunternehmen in Bezug auf die Nutzung von Leiharbeit und enthalten sogar Verpflichtungen im Verhältnis $\mathrm{zu}$ den LeiharbeitnehmerInnen selbst. ${ }^{73}$

\section{Arbeitsvertrag und Arbeitsverhältnis}

Die Suche nach dem „richtigen“ Verantwortlichen für die Arbeitgeberpflichten wird dadurch erschwert, dass der arbeitsrechtliche Schutz im engeren Sinn an der „persönlichen Abhängigkeit“, d.h. an der Einbindung in eine Organisation anknüpft, die durch das Weisungsrecht begründet wird. Denkt man hier konsequent weiter, müsste diese Eingliederung auch den Anknüpfungspunkt für die Arbeitgeberverpflichtungen darstellen. ${ }^{74}$

Die Frage nach der Bedeutung der „Eingliederung“ in den Betrieb als Definitionsmerkmal des Arbeitsverhältnisses hat das Arbeitsrecht von Anfang an begleitet. Mittlerweile ist allerdings unumstritten, dass die Eingliederung allenfalls zusätzlich zum Vertragsschluss eine Rolle spielen kann, nicht jedoch anstelle des Vertragsschlusses. ${ }^{75}$ Denn selbstverständlich muss ein Vertrag die formale Legitimation für die Begründung organisatorischer Herrschaftsverhältnisse bieten.

Leider wurde die Eingliederungstheorie lange Zeit ohne Not mit der Behauptung vermischt, mit dem Arbeitsverhältnis werde ein „Gemeinschaftsverhältnis“ begründet $^{76}$ (eine nichtssagende „romantische Metapher des Organismus“77). Beide Ansätze sind aber unabhängig voneinander. Die Eingliederung in eine hierarchische Organisation ist als soziale Tatsache zu Recht wesentliche Grundlage arbeitsrechtlichen Schutzes (vor allem der betriebsrätlichen Interessenvertretung). Wenn ein Arbeitgeber die Eingliederung von ihrer vertraglichen Grundlage löst und die Arbeitgeberverantwortung in Drittbeziehungen auflöst, spricht viel dafür, jedenfalls diejenigen Aspekte des arbeitsrechtlichen Schutzes unmittelbar an der organisatorischen Einbindung anzuknüpfen, die der einseitigen Herrschaftsmacht der betrieblichen Organisation etwas entgegensetzen; ${ }^{78}$ dies gilt insbesondere für betriebsverfassungsrechtliche Rechte. ${ }^{79}$

Vgl. Estlund, Regoverning the workplace, 2010, $97 \mathrm{ff}$.; kritisch Rieble, BB 2013, $245 \mathrm{ff}$.

Krause, NZA 2012, 830, 835 f; zu den tarifrechtlichen Fragen Krause, AuR 2012, 21; siehe auch Krause, in diesem Heft, S. $119 \mathrm{ff}$.

Vgl. Rechtspolitische Leitlinien der IG Medien aus den 1980er Jahren zu „Arbeitgeber und Betrieb“: „Die Arbeitnehmer in solchen 'abhängigen' Unternehmen gelten in Bezug auf die arbeitsvertraglichen Nebenpflichten auch als Arbeitnehmer des Unternehmens, das für das Arbeitsverhältnis wesentliche Entscheidungen trifft. "(Wendeling-Schröder, in: Festschrift für Albert Gnade, 1992, 367, 373 f.).

Siehe insbesondere den damaligen Hauptvertreter der „Eingliederungstheorie“ Nikisch, RdA 1960, 1 („modifizierte arbeitsrechtliche Eingliederungstheorie“ nach Unterseher, Arbeitsvertrag und innerbetriebliche Herrschaft. Eine historische Untersuchung, 1969, 64 ff., 42 ff.).

Sehr deutlich bei Nikisch (Fn. 78).

Kahn-Freund, Funktionswandel des Arbeitsrechts, 1932, in: Th. Ramm (Hrsg.), Arbeitsrecht und Politik. Quellentexte 1918-1933, 1966, 233 kritisierte so die Rspr. des RAG (dazu sowie weiter kritisch ausführlich Nogler (Fn. 41)).

78 Siehe einen älteren Vorschlag von Heide Pfarr (zitiert bei Wendeling-Schröder (Fn. 77)), 372 f.): „Es wird gesetzlich vermutet, daß Personen, die in einem Betrieb arbeiten, Beschäftigte desjenigen Unternehmens sind, das diesen Betrieb betreibt. Abweichungen bedürfen eines sachlichen Grundes, der nicht in der bloßen Vermeidung von Schutzrechten und arbeitsrechtlichen Ansprüchen liegen darf.“.

Siehe z.B. Karthaus/Klebe (Fn. 5), 420 f. Betriebsbezogenheit und Auslegung nach Zweck der Beteiligungsrechte; vgl. auch Reichold, NZA - Beilage 4/2012, 146, 149: Aufgaben des Betriebsrates („Gestaltungsteilhabe") lassen sich besser organisationsrechtlich als schulärechtlich erklären. 
Erwerbsarbeit kann in unterschiedlichen privatrechtlichen Gestaltungsformen erbracht werden - deren Rechtsfolgen sich vom Arbeitsvertrag über die abhängige Selbstständigkeit bis zu zivilrechtlichen Verträgen ohne arbeitsrechtlichen Schutz gravierend unterscheiden.

Der Unterscheidung zwischen arbeits- und wirtschaftsrechtlich organisierter Beschäftigung liegt eine Vorstellung von grundlegend unterschiedlichen Koordinationsformen der Arbeit zugrunde: Die Wertschöpfung durch Arbeit setzt Koordinationsformen voraus, die arbeitsteilige Prozesse zusammenführen. Diese Koordination kann entweder durch eine Organisation erfolgen oder durch einen Markt. Der Unterscheidung zwischen Organisation und Markt entspricht auf der rechtlichen Seite die Unterscheidung zwischen Arbeitsrecht und Wirtschaftsrecht. Allerdings ist die Grenze zwischen Organisation und Markt nicht (mehr) so scharf, wie es die scharfe Grenzziehung zwischen Arbeits- und Wirtschaftsrecht suggeriert. Je stärker in den Unternehmen Formen der Arbeitskoordination jenseits der klassischen Hierarchien entwickelt werden, desto fließender werden die Übergänge zur marktmäßigen Koordination, d.h. die Abgrenzung zwischen der Vergabe von „Arbeit“ innerhalb einer (hierarchischen) Organisation und dem Einkaufen von Leistungen als „Produkt“ auf einem Markt. Damit aber wird die Organisation der Erwerbsarbeit von Seiten der Auftraggebenden immer stärker disponibel, diese können faktisch zwischen arbeitsund wirtschaftsrechtlichen Formen wählen. ${ }^{80}$

Eine Reaktionsmöglichkeit besteht darin, den rechtlichen Schutz entsprechend zu differenzieren. So schlug der Supiot-Bericht vor, Erwerbsarbeit in vier Rechtskreisen zu regeln: Es seien Regeln zu finden für die abhängige Erwerbsarbeit im engeren Sinn, für die berufliche Arbeit, die nicht-berufliche Arbeit sowie - im äußersten Kreis - für jegliche Tätigkeit unabhängig vom konkreten Beschäftigungsverhältnis. ${ }^{81}$ Im letzten Kreis geht es letztlich um die Festschreibung von „Grundrechten der Erwerbsarbeit“ als eines innersten Kerns der in Person erbrachten Arbeit zur Existenzsicherung. ${ }^{82}$

Zwar ist eingewandt worden, dass die Einführung weiterer Vertrags- und Schutzformen dazu führen könnte, dass die ArbeitgeberInnen künftig noch mehr Ausweich- und Umgehungsmöglichkeiten bekämen. ${ }^{83}$ Allerdings: Diese Ausweichmöglichkeiten gibt es bereits heute, mit dem Unterschied, dass es den zivilrechtlichen Beschäftigungsformen meist an ausreichender sozialer Absicherung mangelt. Um der „commercialization“ ${ }^{84}$ des Arbeitsrechts etwas entgegen zu setzen, bedarf es der Einführung äquivalenter Schutzformen im Zivilrecht. Mark Freedland hat für die Bearbeitung dieser Problematik vor dem Hintergrund des englischen Rechts die Figur des „personal work nexus“ entwickelt; wo es um „personality in work“ geht, müssten Würde, „capability“ und Stabilität rechtlich garantiert sein. ${ }^{85}$ Denn bei allen Verträgen der Erwerbsarbeit geht es darum, dass 
zum Zweck der Existenzsicherung Arbeit in Person und damit mit der Persön-

lichkeit geleistet wird.

Gleichzeitig müssen die „Alleinstellungsmerkmale“ des Arbeitsrechts ernster genommen werden. Die zivilrechtliche Überschätzung des formalen Vertragsschlusses gegenüber den tatsächlichen Organisationsverhältnissen bietet Umgehungsmöglichkeiten, denen eine arbeitsrechtliche Anknüpfung von Arbeitgeberpflichten an der organisatorischen Eingliederung begegnen müsste.

\title{
„Deals“ im Strafprozess Grundlegende Studie über Absprachen
}

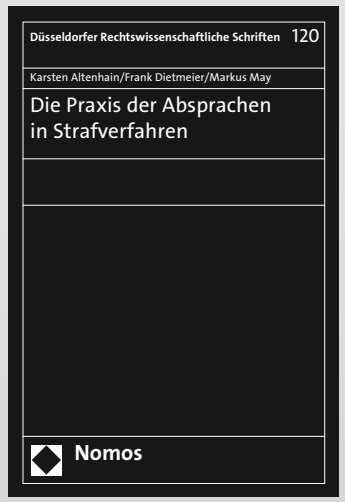

\author{
Die Praxis der Absprachen in \\ Strafverfahren \\ Von Prof. Dr. Karsten Altenhain, \\ Dr. Frank Dietmeier und Markus May \\ 2013, ca. 210 S., brosch., 54,- $€$ \\ ISBN 978-3-8487-0476-7 \\ (Düsseldorfer Rechtswissenschaftliche \\ Schriften, Bd. 120) \\ Erscheint ca. Juni 2013
}

Die empirische Untersuchung, die im Auftrag des Bundesverfassungsgerichts erstellt wurde, kommt zu dem besorgniserregenden Ergebnis, dass die gesetzlichen Vorgaben in der Rechtspraxis weitgehend missachtet werden. Auf dieser Studie beruht auch das aktuelle Grundsatzurteil des BVerfG über „Deals“ im Strafprozess. 